\title{
X-ray spectral components observed in the afterglow of GRB 130925A
}

Bellm, Eric C.; Barriere, Nicolas M.; Bhalerao, Varun; Boggs, Steven E.; Cenko, S. Bradley; Christensen, Finn Erland; Craig, William W.; Forster, Karl; Fryer, Chris L.; Hailey, Charles J.

Total number of authors:

22

Published in:

The Astrophysical Journal Letters

Link to article, DOI:

10.1088/2041-8205/784/2/L19

Publication date:

2014

Document Version

Publisher's PDF, also known as Version of record

Link back to DTU Orbit

Citation (APA):

Bellm, E. C., Barriere, N. M., Bhalerao, V., Boggs, S. E., Cenko, S. B., Christensen, F. E., Craig, W. W., Forster, K., Fryer, C. L., Hailey, C. J., Harrison, F. A., Horesh, A., Kouveliotou, C., Madsen, K. K., Miller, J. M., Ofek, E. O., Perley, D. A., Rana, V. R., Reynolds, S. P., ... Zhang, W. W. (2014). X-ray spectral components observed in the afterglow of GRB 130925A. The Astrophysical Journal Letters, 784(2), [L19]. https://doi.org/10.1088/20418205/784/2/L19

\section{General rights}

Copyright and moral rights for the publications made accessible in the public portal are retained by the authors and/or other copyright owners and it is a condition of accessing publications that users recognise and abide by the legal requirements associated with these rights.

- Users may download and print one copy of any publication from the public portal for the purpose of private study or research.

- You may not further distribute the material or use it for any profit-making activity or commercial gain

- You may freely distribute the URL identifying the publication in the public portal 


\title{
X-RAY SPECTRAL COMPONENTS OBSERVED IN THE AFTERGLOW OF GRB 130925A
}

\author{
Eric C. Bellm ${ }^{1}$, Nicolas M. Barrière ${ }^{2}$, Varun Bhalerao ${ }^{3}$, Steven E. Boggs $^{2}$, S. Bradley Cenko ${ }^{4}$, Finn E. Christensen $^{5}$, \\ William W. Craig ${ }^{2,6}$, Karl Forster ${ }^{1}$, Chris L. Fryer ${ }^{7}$, Charles J. Hailey $^{8}$, Fiona A. Harrison ${ }^{1}$, Assaf Horesh $^{9}$, \\ Chryssa Kouveliotou ${ }^{10}$, Kristin K. Madsen ${ }^{1}$, Jon M. Miller ${ }^{11}$, Eran O. Ofek ${ }^{9}$, Daniel A. Perley ${ }^{1}$, Vikram R. Rana ${ }^{1}$, \\ Stephen P. Reynolds ${ }^{12}$, Daniel Stern ${ }^{13}$, John A. Tomsick ${ }^{2}$, And William W. Zhang ${ }^{4}$ \\ ${ }^{1}$ Cahill Center for Astronomy and Astrophysics, California Institute of Technology, Pasadena, CA 91125, USA; ebellm@ caltech.edu \\ ${ }^{2}$ Space Sciences Laboratory, University of California, Berkeley, CA 94720, USA \\ ${ }^{3}$ Inter-University Center for Astronomy and Astrophysics, Post Bag 4, Ganeshkhind, Pune 411007, India \\ ${ }^{4}$ NASA Goddard Space Flight Center, Greenbelt, MD 20771, USA \\ ${ }^{5}$ DTU Space—National Space Institute, Technical University of Denmark, Elektrovej 327, DK-2800 Lyngby, Denmark \\ ${ }^{6}$ Lawrence Livermore National Laboratory, Livermore, CA 94550, USA \\ ${ }^{7}$ CCS-2, Los Alamos National Laboratory, Los Alamos, NM 87545, USA \\ ${ }^{8}$ Columbia Astrophysics Laboratory, Columbia University, New York, NY 10027, USA \\ ${ }^{9}$ Benoziyo Center for Astrophysics, Weizmann Institute of Science, 76100 Rehovot, Israel \\ ${ }^{10}$ Astrophysics Office/ZP12, NASA Marshall Space Flight Center, Huntsville, AL 35812, USA \\ ${ }^{11}$ Department of Astronomy, The University of Michigan, 500 Church Street, Ann Arbor, MI 48109, USA \\ ${ }^{12}$ Physics Department, NC State University, Raleigh, NC 27695, USA \\ ${ }^{13}$ Jet Propulsion Laboratory, California Institute of Technology, Pasadena, CA 91109, USA \\ Received 2014 January 17; accepted 2014 February 26; published 2014 March 11
}

\begin{abstract}
We have identified spectral features in the late-time X-ray afterglow of the unusually long, slow-decaying GRB 130925A using NuSTAR, Swift/X-Ray Telescope, and Chandra. A spectral component in addition to an absorbed power law is required at $>4 \sigma$ significance, and its spectral shape varies between two observation epochs at $2 \times 10^{5}$ and $10^{6} \mathrm{~s}$ after the burst. Several models can fit this additional component, each with very different physical implications. A broad, resolved Gaussian absorption feature of several $\mathrm{keV}$ width improves the fit, but it is poorly constrained in the second epoch. An additive blackbody or second power-law component provide better fits. Both are challenging to interpret: the blackbody radius is near the scale of a compact remnant $\left(10^{8} \mathrm{~cm}\right)$, while the second power-law component requires an unobserved high-energy cutoff in order to be consistent with the non-detection by Fermi/Large Area Telescope.
\end{abstract}

Key word: gamma-ray burst: individual (GRB 130925A)

Online-only material: color figures

\section{INTRODUCTION}

Recent work has identified several "ultra-long" gamma-ray bursts (GRBs) with properties distinct from normal long GRBs (Levan et al. 2014, and references therein). These events have initial bursting phases lasting thousands of seconds in gammarays and show long-lived, highly variable $\mathrm{X}$-ray afterglows. It is currently unclear whether these bursts are simply extreme examples of the long GRB class, as suggested by Zhang et al. (2013); if they are related to the even longer candidate relativistic tidal disruption events (TDEs) Swift J1644+57 (Bloom et al. 2011; Levan et al. 2011; Burrows et al. 2011) and Swift J2058+05 (Cenko et al. 2012); or if they represent a new subclass of transient, perhaps with large-radius progenitors (Woosley \& Heger 2012; Gendre et al. 2013; Nakauchi et al. 2013).

The bright, nearby GRB 130925A is similar to previously reported ultra-long GRBs and, with the launch of NuSTAR, provides an opportunity to observe the X-ray spectrum at high sensitivity over a broad energy band. Here we report timevarying spectral features in the late-time X-ray afterglow of GRB 130925 A that were initially discovered by NuSTAR and confirmed in a second epoch by NUSTAR and Chandra. Our detections are at higher energies and significantly later times than previously reported afterglow features.

Before the era of routine afterglow observations with Swift/ X-Ray Telescope (XRT), several authors claimed detection of lines in GRB X-ray afterglows on top of otherwise smooth power-law (PL) spectra (e.g., Piro et al. 2000; Amati et al. 2000; Reeves et al. 2002). Most reports were of emission lines at relatively low signal-to-noise ratio $(\mathrm{S} / \mathrm{N})$, and there was substantial controversy over the methods used to assess line significance (Protassov et al. 2002; Sako et al. 2005). Since the advent of Swift, no firm afterglow line detections have been reported despite its greater sensitivity and systematic follow-up, calling previous reports into question (for a review, see Hurkett et al. 2008).

However, statistically significant blackbody components have been reported in the early-time $\left(t \lesssim 10^{3} \mathrm{~s}\right)$ afterglow spectra of several bursts observed by Swift-XRT (Starling et al. 2012 and references therein). The inferred rest-frame temperatures are typically a few tenths of a $\mathrm{keV}$, the inferred radii are $\sim 10^{12} \mathrm{~cm}$, and the blackbody component provides $10 \%-50 \%$ of the $0.3-10 \mathrm{keV}$ flux. The first detections were in low-luminosity, supernova ( $\mathrm{SN}$ )-associated GRBs, leading to suggestions that the emission was due to shock breakout from the SN (e.g., Campana et al. 2006). Systematic searches have found thermal components in early afterglows of classical GRBs as well (Sparre \& Starling 2012; Friis \& Watson 2013), giving credence to alternative interpretations including late-time emission from a prompt photosphere (Friis \& Watson 2013) or emission from a cocoon around the jet (Suzuki \& Shigeyama 2013; Nakauchi et al. 2013).

Of particular relevance are reports of additional components in the afterglows of other ultra-long GRBs. The "Christmas 


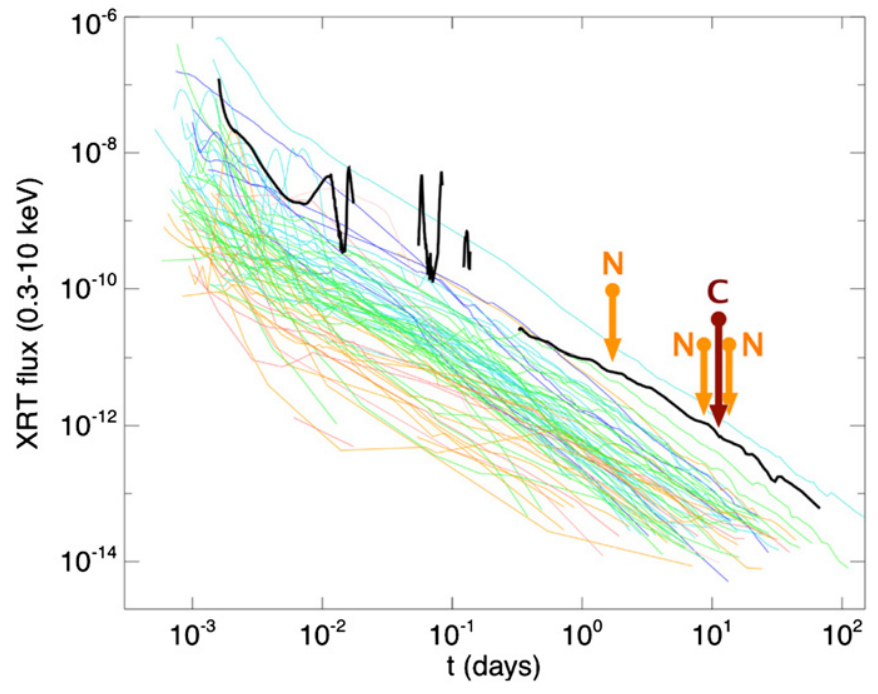

Figure 1. Swift-XRT lightcurve for GRB 130925A (black) plotted over the XRT lightcurves of other afterglows. The NUSTAR (N) and Chandra (C) observation times are marked.

(A color version of this figure is available in the online journal.)

Day Burst" GRB 101225A showed evidence of two separate blackbody components, a $1 \mathrm{keV} \mathrm{X}$-ray blackbody with radius $2 \times 10^{11} \mathrm{~cm}$ observed $6 \mathrm{ks}$ after the burst, and a UVOIR blackbody with radius $2-7 \times 10^{14} \mathrm{~cm}$ which cooled over 18 days (Thöne et al. 2011). In GRB 111209A, Stratta et al. (2013) reported the $X M M$ detection of a second, hard PL component $(\Gamma \sim 0)$ during the steep decay phase $\sim 70 \mathrm{ks}$ after the burst.

\section{OBSERVATIONS}

GRB 130925A produced several emission episodes triggering Swift/Burst Alert Telescope (BAT), Fermi/Gamma-ray Burst Monitor, and MAXI. Swift-BAT triggered on GRB 130925A at $T_{0}=2013-09-25$ 04:11:24 UT (Lien et al. 2013). Fermi-GBM triggered on a precursor episode about 15 minutes before the Swift trigger (Fitzpatrick \& The Fermi GBM Team 2013), and MAXI triggered on an emission episode nearly $4 \mathrm{ks}$ after the initial Swift trigger (Suzuki et al. 2013). The final BAT detection of the emission occurred during a flare observed by XRT, at $T_{0}+7.1 \mathrm{ks}$ (Markwardt et al. 2013). Despite an automated repointing, Fermi/Large Area Telescope (LAT) did not detect any emission (Kocevski et al. 2013). Both the INTEGRAL-SPI Anti-Coincidence Shield and Konus-WIND detected gammarays from the burst over a total interval of nearly $5 \mathrm{ks}$ (Savchenko et al. 2013; Golenetskii et al. 2013).

Swift-XRT observed large, repeated flares from the burst (Evans et al. 2013; Figure 1). The extraordinary length of the bursting phase led Burrows et al. (2013) to suggest similarity to the proposed jetted TDE Swift J1644+57, although Golenetskii et al. (2013) argued that some previous ultra-long events thought to be GRBs had been observed with similar total duration.

Starting around $2 \times 10^{4} \mathrm{~s}$ after the Swift trigger, the X-ray afterglow entered a steady decay phase without new flares (Figure 1). The observed decline is similar to other GRB afterglows and differs markedly from the weeks of flaring observed for Swift J1644+57.

In contrast, the source was faint at optical-NIR wavelengths. Rapid followup observations found a NIR-bright ( $K=18$, $r^{\prime}>22$ mag AB) source near the X-ray position (Sudilovsky et al. 2013a). Spectroscopy of the host galaxy provided a redshift of $z=0.347$ (Vreeswijk et al. 2013; Sudilovsky et al. 2013b). Late-time Hubble Space Telescope imaging showed that the event took place in the plane of a disrupted host galaxy but offset 0 '. 12 (600 pc in projection) from the galaxy nucleus (Tanvir et al. 2013). This offset disfavors a TDE origin for this event, although the authors noted that a galaxy merger could produce a supermassive black hole offset from the light centroid.

NUSTAR (Harrison et al. 2013) provides unprecedented $\mathrm{X}$-ray sensitivity above $10 \mathrm{keV}$ thanks to the combination of its multilayer-coated focusing optics and CdZnTe detectors. NUSTAR observed GRB $130925 \mathrm{~A}$ during the decay phase beginning 1.8 days after the Swift trigger (Figure 1). The total on-source observation time in the first epoch was $39.2 \mathrm{ks}$. Our initial analysis showed that an absorption feature was needed to fit the NuSTAR data (Bellm et al. 2013). We triggered two additional NuSTAR observations of 88.2 and $90.7 \mathrm{ks}$ integration time; these occurred at 8.8 and 11.3 days after the Swift trigger. We also obtained a 44.3 ks Director's Discretionary Time observation with Chandra ACIS-S beginning 11.0 days after the Swift trigger.

\section{DATA REDUCTION}

We processed the NUSTAR data with HEASOFT 6.14 and the NuSTAR Data Analysis Software v.1.2.0 using CALDB version 20130509. We extracted source counts from circular regions with $40^{\prime \prime}$ radius from both $N U S T A R$ modules. We identified background regions of $125^{\prime \prime}$ radius on the same $N u S T A R$ detectors as the source. Since the second and third NuSTAR observations and the Chandra observation are nearly contiguous in time and the source is only slowly varying, we analyzed these data together and refer to them hereafter as the second epoch. We combined the NuSTAR data from the second and third observations and from both modules into a single spectrum to maximize the $S / N$.

We also downloaded and reduced the $13.0 \mathrm{ks}$ of Swift-XRT photon-counting-mode data contemporaneous with the first NUSTAR epoch (obsid 00571830006) using standard procedures in HEASOFT 6.14.

We processed the Chandra data using standard procedures with CIAO v4.5. The data were obtained using $1 / 4$ window readout to reduce pileup; we verified that the effect of pileup on our spectra is negligible and ignore it in further analysis.

We rebinned all of the data to $>20$ counts per bin and fit the data using ISIS v1.6.2-19. We also required the NuSTAR bins to have $\mathrm{S} / \mathrm{N}$ of $>4.5$, as above $\sim 15 \mathrm{keV}$ the background dominates. We minimized $\chi^{2}$ in our fits to the data and use the covariance matrix in our significance calculations in Section 4. We used fit energy bands of 3-30 keV (NuSTAR), 0.3-10 keV (Swift-XRT), and 0.2-10 keV (Chandra). All errors are $90 \%$ confidence limit (C.L.), and we have used a cosmology with $h=0.704, \Omega_{M}=0.273, \Omega_{\Lambda}=0.727$ (Komatsu et al. 2011).

\section{SPECTRAL MODELING}

\subsection{Single Power Law}

GRB X-ray afterglow spectra are usually well-fit by absorbed PL models. We froze a Galactic $N_{\mathrm{H}}$ component of $1.7 \times$ $10^{20} \mathrm{~cm}^{-2}$ (Kalberla et al. 2005; Evans et al. 2013) and allowed a varying $N_{\mathrm{H}}$ component at the reported redshift of $z=0.347$.

A PL fit to the first-epoch NUSTAR data shows a clear deficit in the residuals in the 5-6 keV region (Figure 2). A joint PL fit including the Swift-XRT data improves the parameter constraints, particularly for $N_{\mathrm{H}}$, but the residual structure remains. The goodness of fit is poor, with $\chi_{v}^{2}=1.6$ (Table 1). A PL fit to the 


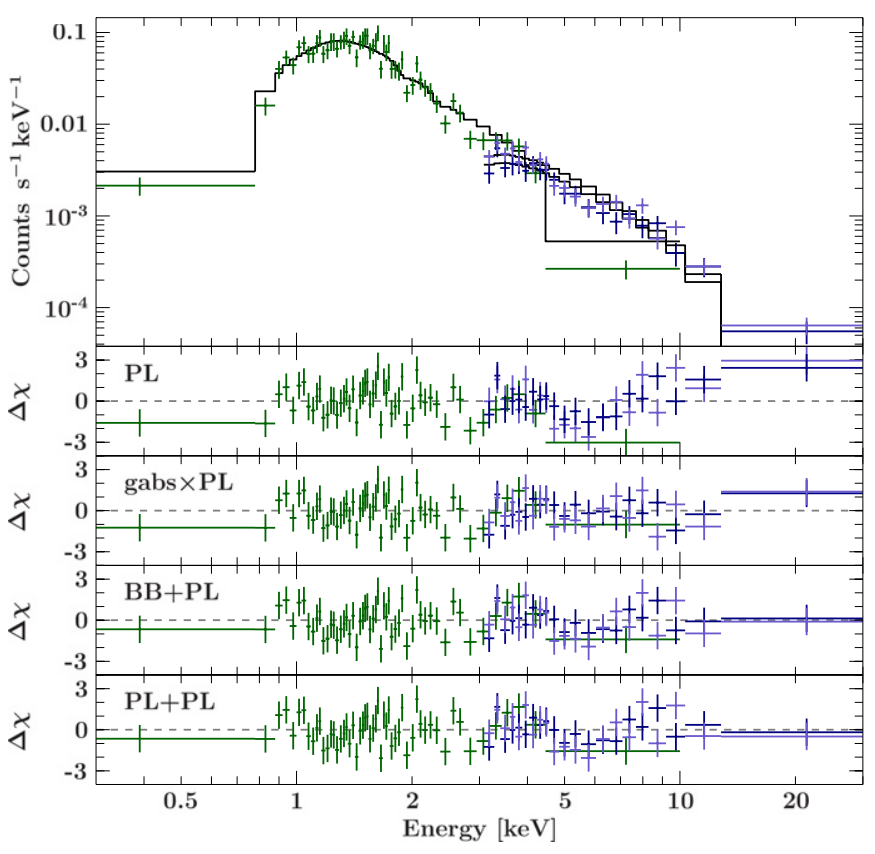

Figure 2. Spectral fits to the first-epoch NUSTAR and Swift data. The top panel shows the count spectra and PL model fit. The lower panels show the residuals for the PL, gabs $\times$ PL, BB + PL, and PL + PL fits. Data are colored blue $(N u S T A R$ module A), navy blue (module B), and green (Swift-XRT).

(A color version of this figure is available in the online journal.)

Chandra data and a joint NuSTAR-Chandra PL fit also show residual structure (Figure 3) and poor goodness of fit, with $\chi_{v}^{2}=2.2$. Additional components (Sections 4.2-4.5) improve these fits.

\subsection{Absorption Feature}

Multiplying by a Gaussian absorber (gabs $\times$ PL) in the first epoch markedly improves the fit residuals relative to a PL fit (Figure 2). The centroid of the Gaussian absorber is at $5.9_{-0.3}^{+0.4} \mathrm{keV}$ and $\sigma=0.9_{-0.3}^{+0.6} \mathrm{keV}$, both in the observer frame. The Swift data show similar residual structure, and in a joint fit the Gaussian absorber gives a similar centroid $\left(6.0_{-0.3}^{+0.5} \mathrm{keV}\right)$ but greater width $\left(1.8_{-0.7}^{+1.9} \mathrm{keV}\right.$; Table 1$)$. In the joint fit, $\chi_{\nu}^{2}$ improves to 1.1 from 1.6 for three additional parameters.

In the second epoch, a Gaussian absorber again improves the fit relative to a PL $\left(\chi_{v}^{2}=1.2\right.$ from 2.2), but the parameters are poorly constrained. The joint NuSTAR and Chandra fit provides only an upper limit $(4.1 \mathrm{keV})$ on the line centroid. This value is inconsistent with that of the first epoch, and the required line width is substantially larger $\left(\sigma=5.2_{-3.0}^{+2.0} \mathrm{keV}\right.$, Figure 4). The large shift in the line centroid is difficult to explain with absorption by a single species. If the large linewidth is interpreted as turbulent velocity broadening, this implies relativistic velocities $\gtrsim 0.1 c$ that increase from the first epoch to the second, an unlikely scenario.

\subsection{Bremsstrahlung}

We obtained good fits $\left(\chi_{v}^{2} \sim 1.1\right)$ with an absorbed bremsstrahlung plus PL model (Bremss + PL). The component is well-constrained in both epochs, with best-fit temperatures of $1.3 \pm 0.2$ and $0.83_{-0.11}^{+0.12} \mathrm{keV}$ in the comoving frame. The fit emission measures are $1.1_{-0.3}^{+0.5} \times 10^{69} \mathrm{~cm}^{-3}$ and $2.3_{-0.6}^{+0.9} \times 10^{68} \mathrm{~cm}^{-3}$. These extreme emission measures, if produced by a constant-density medium, would require densities of order $10^{10}\left(R / 10^{16} \mathrm{~cm}\right)^{-3 / 2} \mathrm{~cm}^{-3}$. However, a circumstellar medium

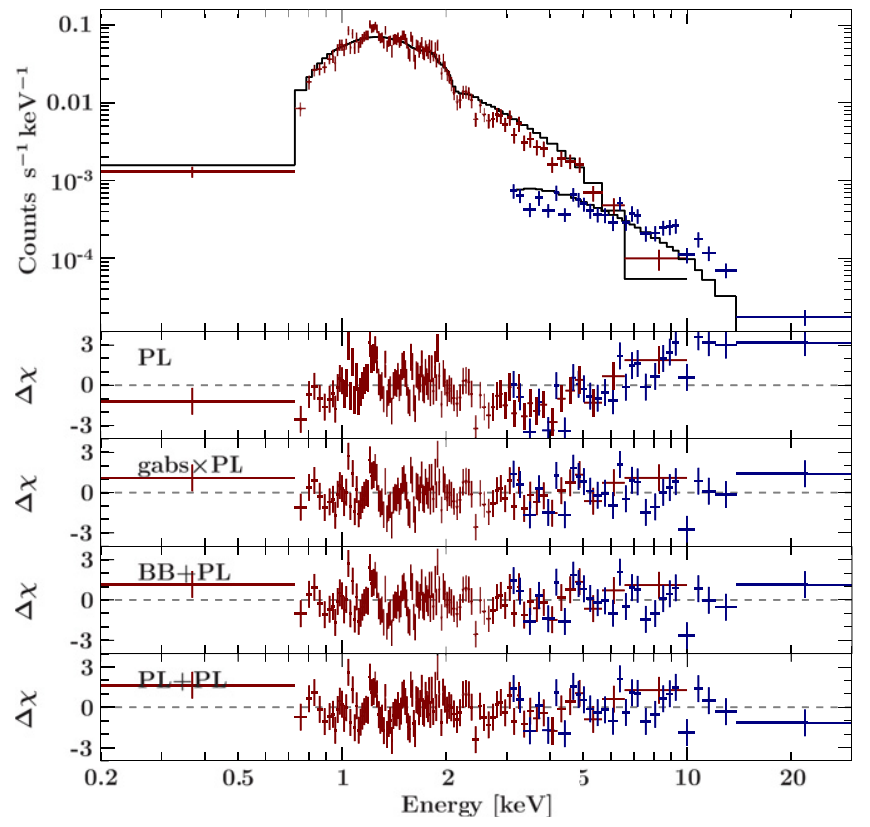

Figure 3. Spectral fits to the second-epoch NUSTAR and Chandra data. Panels are as in Figure 2. The Chandra data are red, and data from the combined NuSTAR modules are navy blue.

(A color version of this figure is available in the online journal.)

this dense would be optically thick to electron scattering, violating the assumptions of the optically thin bremsstrahlung model. The emitting region would be optically thin only if the radius of the region were $>10^{20} \mathrm{~cm}$, much larger than typical afterglow radii. More complex density profiles would require even higher densities at some locations. Thus while the addition of an optically thin bremsstrahlung spectral component improves the fit to the data, we are unable to construct a self-consistent physical interpretation for it. This problem persists even if instead we require a higher temperature for the bremsstrahlung component in order to fit the high-energy excess. The fit is worse ( $\chi^{2}$ increases by 5.9 in both epochs) and provides only a lower limit on the temperature ( $k T \gtrsim 25 \mathrm{keV}$ in the comoving frame). The emission region must still be larger than $10^{18} \mathrm{~cm}$ to be optically thin.

Motivated by the presence of possible additional residual structure in the Chandra data in the $1-3 \mathrm{keV}$ range, we attempted to fit mekal and apec plasma emission models to the second-epoch data. With standard abundances, these models fit metallicity values of zero, reproducing the unphysical Bremss + PL model. Even with highly variable abundances, single-temperature plasmas did not provide clear improvements in the fit.

\subsection{Blackbody}

We also fit a blackbody plus PL model (BB + PL). The $\chi^{2}$ surface shows two minima for the blackbody temperature in both epochs, one near $5 \mathrm{keV}$ and the second near $0.5 \mathrm{keV}$. In the first epoch the higher temperature is preferred $\left(\chi_{\text {low }}^{2}=115.3\right.$ versus $\chi_{\text {high }}^{2}=103.1$ for 90 degrees of freedom (dof)), while in the second epoch the goodness of fit is closer to equivalent $\left(\chi_{\text {low }}^{2}=156.2\right.$ versus $\chi_{\text {high }}^{2}=157.7$ for 130 dof $)$. We argue that the higher-temperature blackbody fit is more plausible due to its relative consistency with the component observed in the first epoch and with theoretical expectations (Section 5).

The blackbody components provide $11 \%$ (29\%) of the total 0.3-30 keV flux in the first (second) epoch. The implied 
Table 1

Best-fit Parameters of Spectral Models

\begin{tabular}{|c|c|c|c|c|c|c|c|c|}
\hline \multirow[t]{2}{*}{ Parameter } & \multicolumn{4}{|c|}{ NuSTAR and Swift Epoch 1} & \multicolumn{4}{|c|}{ NuSTAR and Chandra Epoch 2} \\
\hline & PL & gabs $\times$ PL & $\mathrm{BB}+\mathrm{PL}$ & $P L+P L$ & PL & gabs $\times$ PL & $\mathrm{BB}+\mathrm{PL}$ & $P L+P L$ \\
\hline$N_{\mathrm{H}}\left(10^{22} \mathrm{~cm}^{-2}\right)$ & $2.55_{-0.23}^{+0.24}$ & $2.71_{-0.30}^{+0.41}$ & $3.32_{-0.34}^{+0.35}$ & $3.35_{-0.37}^{+0.40}$ & $1.98 \pm 0.14$ & $2.82_{-0.439}^{+0.31}$ & $2.74_{-0.20}^{+0.21}$ & $3.02_{-0.27}^{+0.29}$ \\
\hline$\Gamma$ & $3.33 \pm 0.13$ & $3.29_{-0.14}^{+0.17}$ & $3.96_{-0.23}^{+0.24}$ & $4.02_{-3.47}^{+0.33}$ & $3.06 \pm 0.11$ & $3.95_{-0.87}^{+0.55}$ & $3.86_{-0.19}^{+0.20}$ & $4.37_{-0.12}^{+0.40}$ \\
\hline$\Gamma_{2}$ & & & & $1.28_{-0.73}^{+0.65}$ & & & & $1.65_{-0.29}^{+0.26}$ \\
\hline$E_{0}(\mathrm{keV})$ & & $5.89_{-0.33}^{+0.53}$ & & & & $<4.09$ & & \\
\hline$\sigma(\mathrm{keV})$ & & $1.75_{-0.70}^{+1.97}$ & & & & $5.15_{-2.97}^{+1.97}$ & & \\
\hline$\tau\left(E=E_{0}\right)$ & & $0.73_{-0.18}^{+0.12}$ & & & & $2.8_{-1.8}^{+1.3}$ & & \\
\hline$k T$ (comoving frame, $\mathrm{keV}$ ) & & & $5.58_{-1.15}^{+2.12}$ & & & & $4.02_{-0.56}^{+0.73}$ & \\
\hline$\chi^{2} / v$ & $146.8 / 92$ & $98.1 / 89$ & $103.1 / 90$ & $105.9 / 90$ & $288.8 / 132$ & $158.9 / 129$ & $157.7 / 130$ & $161.0 / 130$ \\
\hline$P_{\chi}(X>\chi \mid \nu)$ & $2.5 \mathrm{E}-4$ & 0.23 & 0.16 & 0.12 & $1.0 \mathrm{E}-13$ & 0.04 & 0.05 & 0.03 \\
\hline
\end{tabular}

Note. Errors are $90 \%$ C.L.

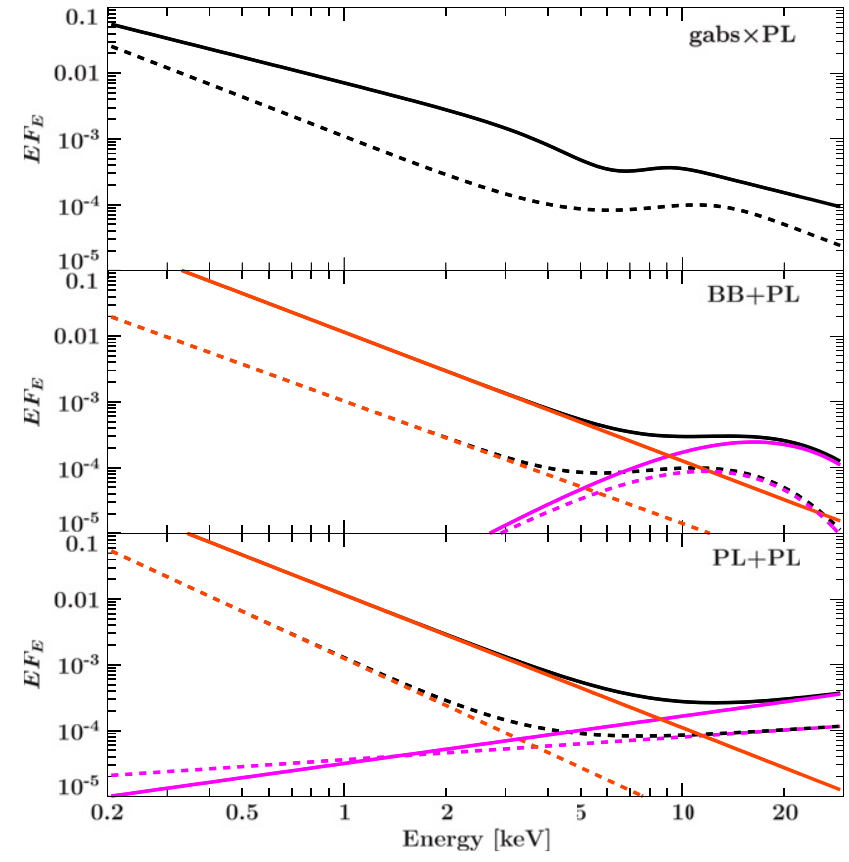

Figure 4. Unfolded, unabsorbed model spectra for the gabs $\times$ PL, BB + PL, and $\mathrm{PL}+\mathrm{PL}$ fits in $\mathrm{keV}^{2} \mathrm{~cm}^{-2} \mathrm{~s}^{-1} \mathrm{keV}^{-1}$. Fits to the first (second) epoch are plotted with solid (dashed) lines. For the $\mathrm{BB}+\mathrm{PL}$ and PL $+\mathrm{PL}$ fits, the total model is plotted in black, the PL component in orange, and the blackbody or second PL component in pink.

(A color version of this figure is available in the online journal.)

radii for a spherical emission region are small and consistent with constant size: $1.1_{-0.8}^{+0.5} \times 10^{8} \mathrm{~cm}$ and $1.5_{-0.6}^{+0.5} \times 10^{8} \mathrm{~cm}$. (The radii for the disfavored low-temperature blackbodies are larger but also relatively compact. However, they imply a physically unlikely contraction of the emitting region from $(3.2 \pm 0.8) \times 10^{10} \mathrm{~cm}$ to $(1.7 \pm 0.4) \times 10^{10} \mathrm{~cm}$. $)$

While blackbody components have been reported in other GRB afterglow spectra, none have been observed at such late times, with such high temperatures, or with such small radii. At 1-10 days after the burst, the blackbody radius inferred from GRB $101225 \mathrm{~A}$ was over $10^{14} \mathrm{~cm}$ and could be explained by the jet interaction with the circumstellar medium (Thöne et al. 2011). The inferred radius of $10^{8} \mathrm{~cm}$ for GRB $130925 \mathrm{~A}$ is much harder to explain with a jet interaction model. This size scale is instead on par with the radius of the fallback accretion disks expected in stellar collapse (Fryer 2009).
If we assume we are observing this disk, the fit temperature can place constraints on the progenitor by constraining the conditions in the disk. The luminosity of an accretion disk is roughly equal to the potential energy released in the accretion. If we consider material at radius $r$, the luminosity $(L)$ is given by $L=G M_{\mathrm{BH}} \dot{m} d r / r^{2}$ where $\dot{m}$ is the accretion rate and $d r$ denotes a small annulus of material at radius $r$ (integrating over $d r$ would produce the total luminosity). The blackbody emission for such an annulus is $L=\sigma A T^{4}=\sigma 2 \pi r d r$, where $\sigma$ is the Stefan-Boltzmann constant and $T$ is the blackbody temperature. If we know the temperature, we can then derive the accretion rate $\dot{m}=\left(2 \pi r^{3} \sigma T^{4}\right) /\left(G M_{\mathrm{BH}}\right)$. For our observed temperatures of 4-5.6 keV, the corresponding accretion rate is $10^{-9}-10^{-10} M_{\odot} \mathrm{s}^{-1}$. Fallback $10^{5}-10^{6} \mathrm{~s}$ after an SN or GRB explosion has been calculated for a range of progenitors and explosion energies (MacFadyen et al. 2001; Wong et al. 2014). Fallback at late times follows a simple PL (Chevalier 1989) and depends on the progenitor and the explosion energy of the SN associated with the GRB. Most fallback calculations (MacFadyen et al. 2001; Wong et al. 2014) predict fallback rates of $10^{-7}-10^{-10} M_{\odot} \mathrm{s}^{-1}$ at $10^{5}-10^{6} \mathrm{~s}$ for SN explosions of $1-3 \times 10^{51} \mathrm{erg}$.

Our accretion rates imply a luminosity near $10^{5}$ times the Eddington limit for a stellar mass black hole. Although such extreme super-Eddington emission rates have been invoked from fallback (Dexter \& Kasen 2013), the exact nature of such transient accretion is not well known. Steady-state solutions of disk accretion find that maintaining emission rates even an order of magnitude above Eddington is difficult (Jaroszynski et al. 1980). Whether such steady-state limits apply in transient situations like our fallback disk remains to be seen (Abramowicz 2005). Thus without a full model of these transient events, we are not able to establish a self-consistent explanation for the blackbody emission.

\subsection{Hard Power Law}

Finally, we considered a two PL model (PL + PL) like that reported for GRB 111209A (Stratta et al. 2013). This model is a slightly worse fit in both epochs than the BB + PL model for the same number of free parameters (Table 1).

Stratta et al. (2013) interpret the very hard $(\Gamma \sim 0)$ second PL component they report for GRB 111209A at $70 \mathrm{ks}$ after the burst as the tail of the hard PL emission sometimes observed by Fermi-LAT (e.g., Zhang et al. 2011 and references therein). This component is detected in the late prompt and 
early afterglow phases and decays according to a PL; its physical origin remains uncertain. The non-detection by LAT of both GRBs complicates this interpretation. An extrapolation of our epoch $1 \mathrm{PL}$ flux to the $0.1-10 \mathrm{GeV}$ band gives a photon flux of $3 \times 10^{-6}$ photons $\mathrm{cm}^{-2} \mathrm{~s}^{-1}$, a value higher than the upper limit of $7 \times 10^{-7}$ photons $\mathrm{cm}^{-2} \mathrm{~s}^{-1}$ reported by Kocevski et al. (2013) in the first $2 \mathrm{ks}$ after the burst, when the afterglow-and thus presumably the hard component-was much brighter. The problem is even more severe for the component reported by Stratta et al. (2013): its higher flux and much harder spectral index extrapolate to a $0.1-10 \mathrm{GeV}$ photon flux of 1.5 photons $\mathrm{cm}^{-2} \mathrm{~s}^{-1}$, an extremely high value sufficient to trigger the LAT. We examined the late-time LAT data for both bursts and confirm no excess emission. Consistency with the nondetection by LAT in both cases thus requires a cutoff above the NuSTAR and XMM bandpasses but below the LAT bandpass at $30 \mathrm{MeV}$. This phenomenological model is plausible, but the connection of these components to the early-time hard PL components detected by LAT in other GRBs therefore remains speculative.

\subsection{Component Significance}

We verified the significance of the additional spectral components using Monte Carlo simulations according to the method of posterior predictive $p$-values (Protassov et al. 2002). We initialized each fit by stepping the additional feature through a grid in energy and finding the largest relative improvement in $\chi^{2}$ (cf. Hurkett et al. 2008). This procedure accounts for the "look-elsewhere" effect of multiple trials, as we have no a priori expectation of the observed line energy or component temperature. In none of our $10^{4}$ simulated realizations of a null PL model did fits with alternative models (gabs $\times$ PL, BB + PL, or $\mathrm{PL}+\mathrm{PL}$ ) produce improvements in $\chi^{2}$ as large as observed in the real data. This implies that the spectral features are significant at $>3.9 \sigma$ in both epochs: the $\chi^{2}$ improvement for each model fit is extremely unlikely to be due to chance if the true underlying model were simply an absorbed PL.

\section{CONCLUSION}

Our late-time afterglow observations of GRB 130925A require an additional spectral component at high significance. Several alternative models provide acceptable fits to the data. These spectral features are detected more than $1 \mathrm{Ms}$ after the burst, much later than any components previously reported in X-ray afterglows, probing a largely unexplored phase of afterglow evolution. Several unique features of GRB 130925A make it possible to detect these late-time features for the first time. The unusually bright afterglow enables high-quality spectral fits, and NuSTAR has excellent sensitivity at the relevant energies and can constrain the continuum above $10 \mathrm{keV}$. Moreover, the primary PL is unusually soft, so the high-energy component is not swamped. It is not yet clear whether this emission is related to progenitor physics unique to this unusual, ultralong burst; NUSTAR observations of the bright "canonical" long GRB 130427A were consistent with emission by a single spectral component (Kouveliotou et al. 2013). Future observations of bright afterglows will be needed to determine the prevalence of these late-time spectral components and identify the relevant emission mechanism.

This work was supported under NASA contract No. NNG08FD60C and uses data from the NuSTAR mission, a project led by the California Institute of Technology, managed by the Jet Propulsion Laboratory, and funded by the National Aeronautics and Space Administration. We thank the NuSTAR Operations team for executing the target of opportunity observations. This research has used the NUSTAR Data Analysis Software (NuSTARDAS) jointly developed by the ASI Science Data Center (ASDC, Italy) and the California Institute of Technology (USA). These results are based in part on observations made by the Chandra X-ray Observatory. We thank the Chandra director for granting discretionary time and the Chandra team for prompt execution of the observations.

Facilities: NuSTAR, Swift, Chandra

\section{REFERENCES}

Abramowicz, M. A. 2005, in Growing Black Holes: Accretion in a Cosmological Context, ed. A. Merloni, S. Nayakshin, \& R. A. Sunyaev (Berlin: Springer), 257

Amati, L., Frontera, F., Vietri, M., et al. 2000, Sci, 290, 953

Bellm, E. C., Harrison, F. A., Forster, K., et al. 2013, GCN, 15286, 1

Bloom, J. S., Giannios, D., Metzger, B. D., et al. 2011, Sci, 333, 203

Burrows, D. N., Kennea, J. A., Ghisellini, G., et al. 2011, Natur, 476, 421

Burrows, D. N., Malesani, D., Lien, A. Y., Cenko, S. B., \& Gehrels, N. 2013, GCN, 15253, 1

Campana, S., Mangano, V., Blustin, A. J., et al. 2006, Natur, 442, 1008

Cenko, S. B., Krimm, H. A., Horesh, A., et al. 2012, ApJ, 753, 77

Chevalier, R. A. 1989, ApJ, 346, 847

Dexter, J., \& Kasen, D. 2013, ApJ, 772, 30

Evans, P. A., Pagani, C., Page, K. L., et al. 2013, GCN, 15254, 1

Fitzpatrick, G. The Fermi GBM Team. 2013, GCN, 15255, 1

Friis, M., \& Watson, D. 2013, ApJ, 771, 15

Fryer, C. L. 2009, ApJ, 699, 409

Gendre, B., Stratta, G., Atteia, J. L., et al. 2013, ApJ, 766, 30

Golenetskii, S., Aptekar, R., Frederiks, D., et al. 2013, GCN, 15260, 1

Harrison, F. A., Craig, W. W., Christensen, F. E., et al. 2013, ApJ, 770, 103

Hurkett, C. P., Vaughan, S., Osborne, J. P., et al. 2008, ApJ, 679, 587

Jaroszynski, M., Abramowicz, M. A., \& Paczynski, B. 1980, AcA, 30, 1

Kalberla, P. M. W., Burton, W. B., Hartmann, D., et al. 2005, A\&A, 440,775

Kocevski, D., Racusin, J., Vianello, G., Axelsson, M., \& Omodei, N. 2013, GCN, 15268, 1

Komatsu, E., Smith, K. M., Dunkley, J., et al. 2011, ApJS, 192, 18

Kouveliotou, C., Granot, J., Racusin, J. L., et al. 2013, ApJL, 779, L1

Levan, A. J., Tanvir, N. R., Cenko, S. B., et al. 2011, Sci, 333, 199

Levan, A. J., Tanvir, N. R., Starling, R. L. C., et al. 2014, ApJ, 781, 13

Lien, A. Y., Markwardt, C. B., Page, K. L., et al. 2013, GCN, 15246, 1

MacFadyen, A. I., Woosley, S. E., \& Heger, A. 2001, ApJ, 550, 410

Markwardt, C. B., Barthelmy, S. D., Baumgartner, W. H., et al. 2013, GCN, 15257,1

Nakauchi, D., Kashiyama, K., Suwa, Y., \& Nakamura, T. 2013, ApJ, 778, 67

Piro, L., Garmire, G., Garcia, M., et al. 2000, Sci, 290, 955

Protassov, R., van Dyk, D. A., Connors, A., Kashyap, V. L., \& Siemiginowska, A. 2002, ApJ, 571, 545

Reeves, J. N., Watson, D., Osborne, J. P., et al. 2002, Natur, 416, 512

Sako, M., Harrison, F. A., \& Rutledge, R. E. 2005, ApJ, 623, 973

Savchenko, V., Beckmann, V., Ferrigno, C., et al. 2013, GCN, 15259, 1

Sparre, M., \& Starling, R. L. C. 2012, MNRAS, 427, 2965

Starling, R. L. C., Page, K. L., Pe'Er, A., Beardmore, A. P., \& Osborne, J. P. 2012, MNRAS, 427, 2950

Stratta, G., Gendre, B., Atteia, J. L., et al. 2013, ApJ, 779, 66

Sudilovsky, V., Kann, D., Greiner, J., et al. (GROND Team) 2013a, GCN, 15247,1

Sudilovsky, V., Kann, D., Schady, P., et al. 2013b, GCN, 15250, 1

Suzuki, A., \& Shigeyama, T. 2013, ApJL, 764, L12

Suzuki, K., Sakakibara, H., Negoro, H., et al. 2013, GCN, 15248, 1

Tanvir, N. R., Levan, A. J., Hounsell, R., et al. 2013, GCN, 15489, 1

Thöne, C. C., de Ugarte Postigo, A., Fryer, C. L., et al. 2011, Natur, 480, 72

Vreeswijk, P. M., Malesani, d., Fynbo, J. P. U., De Cia, A., \& Ledoux, C. 2013, GCN, 15249, 1

Wong, T.-W., Fryer, C. L., Ellinger, C. I., Rockefeller, G., \& Kalogera, V. 2014, arXiv: 1401.3032

Woosley, S. E., \& Heger, A. 2012, ApJ, 752, 32

Zhang, B.-B., Zhang, B., Liang, E.-W., et al. 2011, ApJ, 730, 141

Zhang, B.-B., Zhang, B., Murase, K., Connaughton, V., \& Briggs, M. S. 2013, arXiv: 1310.2540 